\title{
A new fractional model for giving up smoking dynamics
}

\author{
Jagdev Singh ${ }^{1 *}$, Devendra Kumar ${ }^{1}$, Maysaa Al Qurashi ${ }^{2}$ and Dumitru Baleanu ${ }^{3,4}$
}

\author{
"Correspondence: \\ jagdevsinghrathore@gmail.com \\ 'Department of Mathematics, \\ JECRC University, Jaipur, Rajasthan \\ 303905, India \\ Full list of author information is \\ available at the end of the article
}

\begin{abstract}
The key purpose of the present work is to examine a fractional giving up smoking model pertaining to a new fractional derivative with non-singular kernel. The numerical simulations are conducted with the aid of an iterative technique. The existence of the solution is discussed by employing the fixed point postulate, and the uniqueness of the solution is also proved. The effect of various parameters is shown graphically. The numerical results for the smoking model associated with the new fractional derivative are compared with numerical results for a smoking model pertaining to the standard derivative and Caputo fractional derivative.
\end{abstract}

MSC: 34A08; 35A20; 35A22

Keywords: smoking model; fractional differential equations; Caputo-Fabrizio derivative; fixed point postulate; uniqueness

\section{Introduction}

These days, smoking is one of the major health problems in the world. More than 5 million deaths in the world are caused due to the effect of smoking in different organs of human body. A chance of heart attack is $70 \%$ more in smokers compared to the persons who are not smoking. Smokers have a 10\% higher incidence rate of lung cancer than that of nonsmokers. Bad breath, stained teeth, high blood pressure, coughing are the main effects of short-term smoking. In recent years, mouth cancer, throat cancer, lung cancer, gum disease, heart disease, stomach ulcers are the main threatening due to long-term smoking. The life of smokers is 10 to 13 years shorter than that of non-smokers. Smoking kills many individuals in their most active life according to the reports of WHO. Every scientist, doctor and mathematician tries to control smoking for securing the life expectancy of an individual. To give the best representation of cigarette smoking phenomena, mathematicians tried to make different effective smoking models. The different smoking models were proposed by several authors; for example Erturk et al. [1] investigated a giving up smoking model associated with the Caputo fractional derivative, Zaman [2] analyzed the optimal campaign in the smoking dynamical system, Zaman [3] studied the qualitative response of dynamics of giving up smoking, Lubin and Caporaso [4] discussed cigarette smoking and lung cancer, Garsow et al. [5] examined the mathematical description of the dynamics of tobacco use, recovery and relapse, Sharomi and Gumel [6] demonstrated the curtailing smoking dynamics, Zeb et al. [7] investigated a fractional giving up smoking

(c) The Author(s) 2017. This article is distributed under the terms of the Creative Commons Attribution 4.0 International License (http://creativecommons.org/licenses/by/4.0/), which permits unrestricted use, distribution, and reproduction in any medium, provided you give appropriate credit to the original author(s) and the source, provide a link to the Creative Commons license, and indicate if changes were made. 
model, Alkhudhari et al. [8] analyzed the global dynamics of mathematical equations describing smoking, Khalid et al. [9] explained the fractional mathematical model of giving up smoking and many others.

Fractional calculus is applied in different directions of physics, mathematical biology, fluid mechanics, electrochemistry, signal processing, viscoelasticity, finance and in many more. In the branch of fractional calculus, fractional derivatives and fractional integrals are important aspects. Recently, many researchers and scientists have analyzed issues in this special branch [10-22]. Caputo [10] introduced a fractional derivative which allows the conventional initial and boundary conditions associated with the real world problem. Baleanu et al. [11] reported new advances in nanotechnology and fractional calculus and related issues in their monograph. Kilbas et al. [12] presented basic concepts of fractional differential equations and their applications. Bulut et al. [13] studied the differential equations of arbitrary order by making use of analytical techniques. Atangana and Alkahtani [14] examined the Keller-Segel model pertaining to a fractional derivative having nonsingular kernel. Atangana and Alkahtani [15] studied a fractional non-homogeneous heat model. Singh et al. [16] explained a fractional biological population model. Kumar et al. [17] analyzed the local fractional Klein-Gordon equations. Singh et al. [18] investigated fractional coupled Burgers equations. Kumar et al. [19] presented the numerical solution of a differential-difference equation of arbitrary order having applications in nanotechnology. Area et al. [20] studied the fractional order ebola epidemic model. Carvalho and Pinto [21] analyzed a delay fractional order model for the co-infection of malaria and HIV/AIDS. Huang et al. [22] studied a novel use of the fractional logistic map. Ma et al. [23] reported new results for multidimensional diffusion equations pertaining to local fractional derivative. Kumar et al. [24] analyzed a logistic equation involving a new fractional derivative having a non-singular kernel. Kumar et al. [25] studied a modified Kawahara equation pertaining to a fractional derivative with non-singular kernel.

In a very recent attempt, Caputo and Fabrizio [26] propounded a novel fractional derivative having exponential kernel and in addition Losada and Nieto [27] analyzed the properties of a newly presented fractional derivative. The classical fractional derivatives, especially the Caputo and Riemann derivatives, have their own limitation because their kernel is singular. Since the kernel is employed to describe the memory effect of the physical system, it is obvious that due to this weakness, both derivatives cannot precisely describe the full effect of the memory. Therefore, we use the novel Caputo-Fabrizio (CF) fractional derivative to study the giving up smoking model and explain this problem in a better and more efficient manner.

The key objective of this work is to use the new fractional derivative in the giving up smoking model and imparting the details of the exactness and uniqueness of the solution by applying the fixed point theorem. The development of this article is as follows. In Section 2, the CF fractional order derivative is discussed. In Section 3, the fractional smoking model and approximate solution pertaining to novel CF fractional derivative is discussed. In Section 4, the existence and uniqueness of system of solutions is proved with the aid of the fixed point theorem. Results and discussion are given in Section 5. Lastly in Section 6, the concluding remarks are presented.

\section{Preliminaries}

In the present part, we give some definitions and properties of the fractional derivative as suggested by Caputo and Fabrizio [26]. 
Definition 2.1 Suppose $g \in H^{1}(a, b), b>a, \rho \in[0,1]$; then the newly presented Caputo derivative of arbitrary order [26] is given by

$$
D_{t}^{\rho}(g(t))=\frac{M(\rho)}{1-\rho} \int_{a}^{t} g^{\prime}(x) \exp \left[-\rho \frac{t-x}{1-\rho}\right] d x
$$

where $M(\rho)$ is the normalization of the function satisfying the condition $M(0)=M(1)=1$ [26].

But, if $g \notin H^{1}(a, b)$ under this condition, the derivative is given follows:

$$
D_{t}^{\rho}(g(t))=\frac{\rho M(\rho)}{1-\rho} \int_{a}^{t}(g(t)-g(x)) \exp \left[-\rho \frac{t-x}{1-\rho}\right] d x .
$$

Remark 1 If $\sigma=\frac{1-\rho}{\rho} \in[0, \infty), \rho=\frac{1}{1+\sigma} \in[0,1]$, then equation (2) assumes the form

$$
D_{t}^{\rho}(g(t))=\frac{N(\sigma)}{\sigma} \int_{a}^{t} g^{\prime}(x) \exp \left[-\frac{t-x}{\sigma}\right] d x, \quad N(0)=N(\infty)=1 .
$$

Moreover,

$$
\lim _{\sigma \rightarrow 0} \frac{1}{\sigma} \exp \left[-\frac{t-x}{\sigma}\right]=\delta(x-t)
$$

It is essential to have the anti-derivative associated with the new fractional derivative [27].

Definition 2.2 Let $0<\rho<1$. If $g$ is a function, then the fractional integral of order $\rho$ is presented by

$$
I_{t}^{\rho}(g(t))=\frac{2(1-\rho)}{(2-\rho) M(\rho)} g(t)+\frac{2 \rho}{(2-\rho) M(\rho)} \int_{0}^{t} g(s) d s, \quad t \geq 0 .
$$

Furthermore, the following result holds [27]:

$$
\frac{2(1-\rho)}{(2-\rho) M(\rho)}+\frac{2 \rho}{(2-\rho) M(\rho)}=1
$$

which yields $M(\rho)=\frac{2}{2-\rho}, 0 \leq \rho \leq 1$.

In view of the above results, the authors of [27] have given another form of the newly presented Caputo derivative for the order $0<\rho<1$ represented as

$$
D_{t}^{\rho}(g(t))=\frac{1}{1-\rho} \int_{0}^{t} g^{\prime}(x) \exp \left[-\rho \frac{t-x}{1-\rho}\right] d x, \quad t \geq 0
$$

\section{Model description and giving up smoking model with a fractional derivative involving non-singular kernel}

In this section, we examine the giving up smoking dynamics discussed by Erturk et al. [1]. Suppose the overall population at time $t$ is denoted by $T(t)$. We separate the population $T(t)$ into five subgroups, potential smokers $P(t)$, occasional smokers $L(t)$, heavy smokers 
$S(t)$, temporary quitters $Q(t)$ and smokers who quit permanently $R(t)$ specified by $T(t)=$ $P(t)+L(t)+S(t)+Q(t)+R(t)$. The model is developed as follows:

$$
\begin{aligned}
& \frac{d P}{d t}=a(1-P)-b P S, \\
& \frac{d L}{d t}=-a L+b P L-c L S, \\
& \frac{d S}{d t}=-(a+d) S+c L S+f Q, \\
& \frac{d Q}{d t}=-(a+f) Q+d(1-e) S, \\
& \frac{d R}{d t}=-a R+e d S .
\end{aligned}
$$

In the above system (8) $b$ indicates the contact rate between smokers who smoke occasionally and potential smokers, $a$ denotes the rate of natural death, $c$ stands for the contact rate between smokers who smoke occasionally and temporary quitters, $f$ represents the contact rate between temporary quitters who return back to smoking and smokers, $d$ indicates the rate of giving up smoking, $(1-e)$ stands for the fraction of smokers who temporarily give up smoking (at a rate $d$ ), $e$ denotes the remaining fraction of smokers who give up smoking forever (at a rate $d$ ).

Thus, in order to introduce the above effect into the mathematical representation, we moderate the system by substituting the time-derivative by the newly introduced CaputoFabrizio arbitrarily ordered derivative [26] as given by

$$
\begin{aligned}
& { }_{0}{ }_{0} D_{t}^{\rho} P=a(1-P)-b P S, \\
& { }_{0}^{\mathrm{CF}} D_{t}^{\rho} L=-a L+b P L-c L S, \\
& { }_{0}^{\mathrm{CF}} D_{t}^{\rho} S=-(a+d) S+c L S+f Q, \\
& { }_{0}^{\mathrm{CF}} D_{t}^{\rho} Q=-(a+f) Q+d(1-e) S, \\
& { }_{0}^{{ }_{0}} D_{t}^{\rho} R=-a R+e d S .
\end{aligned}
$$

These come with the initial conditions

$$
P(0)=\delta_{1}, \quad L(0)=\delta_{2}, \quad S(0)=\delta_{3}, \quad Q(0)=\delta_{4} \quad \text { and } \quad R(0)=\delta_{5} .
$$

\section{Existence and uniqueness of a system of solutions of smoking model}

Since our main goal is to investigate the numerical results predicted by the fractional model based on the CF derivative we firstly start to investigate the existence and uniqueness of the solutions. We examine the existence of the system of solutions by applying the fixed point theorem. Employing the fractional integral operator due to Nieto and Losada [27] on equation (9), we obtain

$$
\begin{aligned}
& P(t)-P(0)={ }_{0}^{\mathrm{C}} I_{t}^{\rho}\{a(1-P)-b P S\}, \\
& L(t)-L(0)={ }_{0}^{\mathrm{CF}} I_{t}^{\rho}\{-a L+b P L-c L S\}, \\
& S(t)-S(0)={ }_{0}^{\mathrm{CF}} I_{t}^{\rho}\{-(a+d) S+c L S+f Q\},
\end{aligned}
$$




$$
\begin{aligned}
& Q(t)-Q(0)={ }_{0}^{\mathrm{CF}} I_{t}^{\rho}\{-(a+f) Q+d(1-e) S\}, \\
& R(t)-R(0)={ }_{0}^{\mathrm{CF}} I_{t}^{\rho}\{-a R+e d S\} .
\end{aligned}
$$

By using the notation presented by Nieto and Losada [27], we get

$$
\begin{aligned}
P(t)-P(0)= & \frac{2(1-\rho)}{(2-\rho) M(\rho)}\{a(1-P(t))-b P(t) S(t)\} \\
& +\frac{2 \rho}{(2-\rho) M(\rho)} \int_{0}^{t}\{a(1-P(y))-b P(y) S(y)\} d y, \\
L(t)-L(0)= & \frac{2(1-\rho)}{(2-\rho) M(\rho)}\{-a L(t)+b P(t) L(t)-c L(t) S(t)\} \\
& +\frac{2 \rho}{(2-\rho) M(\rho)} \int_{0}^{t}\{-a L(y)+b P(y) L(y)-c L(y) S(y)\} d y, \\
S(t)-S(0)= & \frac{2(1-\rho)}{(2-\rho) M(\rho)}\{-(a+d) S(t)+c L(t) S(t)+f Q(t)\} \\
& +\frac{2 \rho}{(2-\rho) M(\rho)} \int_{0}^{t}\{-(a+d) S(y)+c L(y) S(y)+f Q(y)\} d y, \\
Q(t)-Q(0)= & \frac{2(1-\rho)}{(2-\rho) M(\rho)}\{-(a+f) Q(t)+d(1-e) S(t)\} \\
& +\frac{2 \rho}{(2-\rho) M(\rho)} \int_{0}^{t}\{-(a+f) Q(y)+d(1-e) S(y)\} d y, \\
& +\frac{2(1-\rho)}{(2-\rho) M(\rho)}\{-a R(t)+e d S(t)\} \\
R(t)-R(0)= & \frac{2 \rho}{(2-\rho) M(\rho)}\{-a R(y)+e d S(y)\} d y .
\end{aligned}
$$

For clarity, we write

$$
\begin{aligned}
& K_{1}(t, P)=a(1-P(t))-b P(t) S(t), \\
& K_{2}(t, L)=-a L(t)+b P(t) L(t)-c L(t) S(t), \\
& K_{3}(t, S)=-(a+d) S(t)+c L(t) S(t)+f Q(t), \\
& K_{4}(t, Q)=-(a+f) Q(t)+d(1-e) S(t), \\
& K_{5}(t, R)=-a R(t)+e d S(t) .
\end{aligned}
$$

Theorem 4.1 The kernels $K_{1}, K_{2}, K_{3}, K_{4}$ and $K_{5}$ satisfy the Lipschitz condition and contraction if the following inequality holds:

$$
0<a+\beta b \leq 1
$$

Proof We start with the kernel $K_{1}$. Let $P$ and $P_{1}$ be two functions, then we assess the following:

$$
\left\|K_{1}(t, P)-K_{1}\left(t, P_{1}\right)\right\|=\left\|-a\left\{P(t)-P_{1}(t)\right\}-b\left\{P(t)-P_{1}(t)\right\} S(t)\right\| .
$$


By using the triangular inequality on equation (14), we have

$$
\begin{aligned}
\left\|K_{1}(t, P)-K_{1}\left(t, P_{1}\right)\right\| & \leq\left\|a\left\{P(t)-P_{1}(t)\right\}\right\|+\left\|b\left\{P(t)-P_{1}(t)\right\} S(t)\right\| \\
& \leq\{a+b\|S(t)\|\}\left\|P(t)-P_{1}(t)\right\| \\
& \leq\{a+b \beta\}\left\|P(t)-P_{1}(t)\right\| \leq \gamma_{1}\left\|\left(P(t)-P_{1}(t)\right)\right\| .
\end{aligned}
$$

Taking $\gamma_{1}=a+\beta b$ where the $\beta=S(t)$ are bounded functions, then we get

$$
\left\|K_{1}(t, P)-K_{1}\left(t, P_{1}\right)\right\| \leq \gamma_{1}\left\|P(t)-P_{1}(t)\right\|
$$

Hence, the Lipschitz condition is satisfied for $K_{1}$, and if additionally $0<(a+\beta b) \leq 1$, then it is also a contraction for $K_{1}$.

Similarly the second, third, fourth and fifth cases satisfy the Lipschitz condition given as follows:

$$
\begin{aligned}
& \left\|K_{2}(t, L)-K_{2}\left(t, L_{1}\right)\right\| \leq \gamma_{2}\left\|L(t)-L_{1}(t)\right\|, \\
& \left\|K_{3}(t, S)-K_{3}\left(t, S_{1}\right)\right\| \leq \gamma_{3}\left\|S(t)-S_{1}(t)\right\|, \\
& \left\|K_{4}(t, Q)-K_{4}\left(t, Q_{1}\right)\right\| \leq \gamma_{4}\left\|Q(t)-Q_{1}(t)\right\|, \\
& \left\|K_{5}(t, R)-K_{5}\left(t, R_{1}\right)\right\| \leq \gamma_{5}\left\|R(t)-R_{1}(t)\right\| .
\end{aligned}
$$

On consideration of the aforesaid kernels, equation (12) becomes

$$
\begin{aligned}
& P(t)=P(0)+\frac{2(1-\rho)}{(2-\rho) M(\rho)} K_{1}(t, P)+\frac{2 \rho}{(2-\rho) M(\rho)} \int_{0}^{t}\left(K_{1}(y, P)\right) d y, \\
& L(t)=L(0)+\frac{2(1-\rho)}{(2-\rho) M(\rho)} K_{2}(t, L)+\frac{2 \rho}{(2-\rho) M(\rho)} \int_{0}^{t}\left(K_{2}(y, L)\right) d y, \\
& S(t)=S(0)+\frac{2(1-\rho)}{(2-\rho) M(\rho)} K_{3}(t, S)+\frac{2 \rho}{(2-\rho) M(\rho)} \int_{0}^{t}\left(K_{3}(y, S)\right) d y, \\
& Q(t)=Q(0)+\frac{2(1-\rho)}{(2-\rho) M(\rho)} K_{4}(t, Q)+\frac{2 \rho}{(2-\rho) M(\rho)} \int_{0}^{t}\left(K_{4}(y, Q)\right) d y, \\
& R(t)=R(0)+\frac{2(1-\rho)}{(2-\rho) M(\rho)} K_{5}(t, R)+\frac{2 \rho}{(2-\rho) M(\rho)} \int_{0}^{t}\left(K_{5}(y, R)\right) d y .
\end{aligned}
$$

Now, we present the following recursive formula:

$$
\begin{aligned}
& P_{n}(t)=\frac{2(1-\rho)}{(2-\rho) M(\rho)} K_{1}\left(t, P_{n-1}\right)+\frac{2 \rho}{(2-\rho) M(\rho)} \int_{0}^{t}\left(K_{1}\left(y, P_{n-1}\right)\right) d y, \\
& L_{n}(t)=\frac{2(1-\rho)}{(2-\rho) M(\rho)} K_{2}\left(t, L_{n-1}\right)+\frac{2 \rho}{(2-\rho) M(\rho)} \int_{0}^{t}\left(K_{2}\left(y, L_{n-1}\right)\right) d y, \\
& S_{n}(t)=\frac{2(1-\rho)}{(2-\rho) M(\rho)} K_{3}\left(t, S_{n-1}\right)+\frac{2 \rho}{(2-\rho) M(\rho)} \int_{0}^{t}\left(K_{3}\left(y, S_{n-1}\right)\right) d y, \\
& Q_{n}(t)=\frac{2(1-\rho)}{(2-\rho) M(\rho)} K_{4}\left(t, Q_{n-1}\right)+\frac{2 \rho}{(2-\rho) M(\rho)} \int_{0}^{t}\left(K_{4}\left(y, Q_{n-1}\right)\right) d y, \\
& R_{n}(t)=\frac{2(1-\rho)}{(2-\rho) M(\rho)} K_{5}\left(t, R_{n-1}\right)+\frac{2 \rho}{(2-\rho) M(\rho)} \int_{0}^{t}\left(K_{5}\left(y, R_{n-1}\right)\right) d y .
\end{aligned}
$$


The initial conditions are given as follows:

$$
\begin{aligned}
& P_{0}(t)=P(0), \\
& L_{0}(t)=L(0), \\
& S_{0}(t)=S(0), \\
& Q_{0}(t)=Q(0), \\
& R_{0}(t)=R(0) .
\end{aligned}
$$

Now, we present the difference between the successive terms in the following manner:

$$
\begin{aligned}
\phi_{n}(t)= & P_{n}(t)-P_{n-1}(t)=\frac{2(1-\rho)}{(2-\rho) M(\rho)}\left(K_{1}\left(t, P_{n-1}\right)-K_{1}\left(t, P_{n-2}\right)\right) \\
& +\frac{2 \rho}{(2-\rho) M(\rho)} \int_{0}^{t}\left(K_{1}\left(y, P_{n-1}\right)-K_{1}\left(y, P_{n-2}\right)\right) d y, \\
\psi_{n}(t)= & L_{n}(t)-L_{n-1}(t)=\frac{2(1-\rho)}{(2-\rho) M(\rho)}\left(K_{2}\left(t, L_{n-1}\right)-K_{2}\left(t, L_{n-2}\right)\right) \\
& +\frac{2 \rho}{(2-\rho) M(\rho)} \int_{0}^{t}\left(K_{2}\left(y, L_{n-1}\right)-K_{2}\left(y, L_{n-2}\right)\right) d y, \\
\xi_{n}(t)= & S_{n}(t)-S_{n-1}(t)=\frac{2(1-\rho)}{(2-\rho) M(\rho)}\left(K_{3}\left(t, S_{n-1}\right)-K_{3}\left(t, S_{n-2}\right)\right) \\
& +\frac{2 \rho}{(2-\rho) M(\rho)} \int_{0}^{t}\left(K_{3}\left(y, S_{n-1}\right)-K_{3}\left(y, S_{n-2}\right)\right), \\
\chi_{n}(t)= & Q_{n}(t)-Q_{n-1}(t)=\frac{2(1-\rho)}{(2-\rho) M(\rho)}\left(K_{4}\left(t, Q_{n-1}\right)-K_{4}\left(t, Q_{n-2}\right)\right) \\
& +\frac{2 \rho}{(2-\rho) M(\rho)} \int_{0}^{t}\left(K_{4}\left(y, Q_{n-1}\right)-K_{4}\left(y, Q_{n-2}\right)\right) d y, \\
S_{n}(t)= & R_{n}(t)-R_{n-1}(t)=\frac{2(1-\rho)}{(2-\rho) M(\rho)}\left(K_{5}\left(t, R_{n-1}\right)-K_{5}\left(t, R_{n-2}\right)\right) \\
& +\frac{2 \rho}{(2-\rho) M(\rho)} \int_{0}^{t}\left(K_{5}\left(y, R_{n-1}\right)-K_{5}\left(y, R_{n-2}\right)\right) d y .
\end{aligned}
$$

It is worth noticing that

$$
\begin{aligned}
& P_{n}(t)=\sum_{i=0}^{n} \phi_{i}(t), \\
& L_{n}(t)=\sum_{i=0}^{n} \psi_{i}(t), \\
& S_{n}(t)=\sum_{i=0}^{n} \xi_{i}(t), \\
& Q_{n}(t)=\sum_{i=0}^{n} \chi_{i}(t), \\
& R_{n}(t)=\sum_{i=0}^{n} \varsigma_{i}(t) .
\end{aligned}
$$


Step by step but surely we assess

$$
\begin{aligned}
\left\|\phi_{n}(t)\right\|= & \left\|P_{n}(t)-P_{n-1}(t)\right\| \\
= & \| \frac{2(1-\rho)}{(2-\rho) M(\rho)}\left(K_{1}\left(t, P_{n-1}\right)-K_{1}\left(t, P_{n-2}\right)\right) \\
& +\frac{2 \rho}{(2-\rho) M(\rho)} \int_{0}^{t}\left(K_{1}\left(y, P_{n-1}\right)-K_{1}\left(y, P_{n-2}\right)\right) d y \| .
\end{aligned}
$$

Employing the triangular inequality, equation (23) reduces to

$$
\begin{aligned}
\left\|P_{n}(t)-P_{n-1}(t)\right\| \leq & \frac{2(1-\rho)}{(2-\rho) M(\rho)}\left\|\left(K_{1}\left(t, P_{n-1}\right)-K_{1}\left(t, P_{n-2}\right)\right)\right\| \\
& +\frac{2 \rho}{(2-\rho) M(\rho)}\left\|\int_{0}^{t}\left(K_{1}\left(y, P_{n-1}\right)-K_{1}\left(y, P_{n-2}\right)\right) d y\right\| .
\end{aligned}
$$

As the kernel satisfies the Lipchitz condition, we have

$$
\begin{aligned}
\left\|P_{n}(t)-P_{n-1}(t)\right\| \leq & \frac{2(1-\rho)}{(2-\rho) M(\rho)} \gamma_{1}\left\|P_{n-1}-P_{n-2}\right\| \\
& +\frac{2 \rho}{(2-\rho) M(\rho)} \gamma_{1} \int_{0}^{t}\left\|P_{n-1}-P_{n-2}\right\| d y,
\end{aligned}
$$

then we get

$$
\left\|\phi_{n}(t)\right\| \leq \frac{2(1-\rho)}{(2-\rho) M(\rho)} \gamma_{1}\left\|\phi_{n-1}(t)\right\|+\frac{2 \rho}{(2-\rho) M(\rho)} \gamma_{1} \int_{0}^{t}\left\|\phi_{n-1}(y)\right\| d y .
$$

Similarly, we get the following results:

$$
\begin{aligned}
\left\|\psi_{n}(t)\right\| & \leq \frac{2(1-\rho)}{(2-\rho) M(\rho)} \gamma_{2}\left\|\psi_{n-1}(t)\right\|+\frac{2 \rho}{(2-\rho) M(\rho)} \gamma_{2} \int_{0}^{t}\left\|\psi_{n-1}(y)\right\| d y, \\
\left\|\xi_{n}(t)\right\| & \leq \frac{2(1-\rho)}{(2-\rho) M(\rho)} \gamma_{3}\left\|\xi_{n-1}(t)\right\|+\frac{2 \rho}{(2-\rho) M(\rho)} \gamma_{3} \int_{0}^{t}\left\|\xi_{n-1}(y)\right\| d y, \\
\left\|\chi_{n}(t)\right\| & \leq \frac{2(1-\rho)}{(2-\rho) M(\rho)} \gamma_{4}\left\|\chi_{n-1}(t)\right\|+\frac{2 \rho}{(2-\rho) M(\rho)} \gamma_{4} \int_{0}^{t}\left\|\chi_{n-1}(y)\right\| d y, \\
\left\|\zeta_{n}(t)\right\| & \leq \frac{2(1-\rho)}{(2-\rho) M(\rho)} \gamma_{5}\left\|\varsigma_{n-1}(t)\right\|+\frac{2 \rho}{(2-\rho) M(\rho)} \gamma_{5} \int_{0}^{t}\left\|\varsigma_{n-1}(y)\right\| d y .
\end{aligned}
$$

On consideration of the above results, we may present the subsequent theorem.

Theorem 4.2 The fractional giving up smoking model (9) has a system of solutions under the conditions that we can find $t_{0}$ such that

$$
\frac{2(1-\rho)}{(2-\rho) M(\rho)} \gamma_{1}+\frac{2 \rho}{(2-\rho) M(\rho)} \gamma_{1} t_{0} \leq 1
$$

Proof Here we have considered that the functions $P(t), L(t), S(t), Q(t)$ and $R(t)$ are bounded. Additionally, we have proven that the kernels satisfy the Lipschitz condition, 
hence on consideration of the results of equation (26) and equation (27) and by employing the recursive method, we derive the succeeding relation as follows:

$$
\begin{aligned}
& \left\|\phi_{n}(t)\right\| \leq\|P(0)\|\left[\left(\frac{2(1-\rho)}{(2-\rho) M(\rho)} \gamma_{1}\right)+\left(\frac{2 \rho}{(2-\rho) M(\rho)} \gamma_{1} t\right)\right]^{n}, \\
& \left\|\psi_{n}(t)\right\| \leq\|L(0)\|\left[\left(\frac{2(1-\rho)}{(2-\rho) M(\rho)} \gamma_{2}\right)+\left(\frac{2 \rho}{(2-\rho) M(\rho)} \gamma_{2} t\right)\right]^{n}, \\
& \left\|\xi_{n}(t)\right\| \leq\|S(0)\|\left[\left(\frac{2(1-\rho)}{(2-\rho) M(\rho)} \gamma_{3}\right)+\left(\frac{2 \rho}{(2-\rho) M(\rho)} \gamma_{3} t\right)\right]^{n}, \\
& \left\|\varphi_{n}(t)\right\| \leq\|Q(0)\|\left[\left(\frac{2(1-\rho)}{(2-\rho) M(\rho)} \gamma_{4}\right)+\left(\frac{2 \rho}{(2-\rho) M(\rho)} \gamma_{4} t\right)\right]^{n}, \\
& \left\|S_{n}(t)\right\| \leq\|R(0)\|\left[\left(\frac{2(1-\rho)}{(2-\rho) M(\rho)} \gamma_{5}\right)+\left(\frac{2 \rho}{(2-\rho) M(\rho)} \gamma_{5} t\right)\right]^{n} .
\end{aligned}
$$

Therefore, the system of functions (22) exists and is smooth. However, to show that the above functions are a system of solutions of the system of equations (9), we assume

$$
\begin{aligned}
& P(t)-P(0)=P_{n}(t)-B_{n}(t), \\
& L(t)-L(0)=L_{n}(t)-C_{n}(t), \\
& S(t)-S(0)=S_{n}(t)-D_{n}(t), \\
& Q(t)-Q(0)=Q_{n}(t)-E_{n}(t), \\
& R(t)-R(0)=R_{n}(t)-F_{n}(t) .
\end{aligned}
$$

Therefore, we have

$$
\begin{aligned}
\left\|B_{n}(t)\right\|= & \| \frac{2(1-\rho)}{(2-\rho) M(\rho)}\left(K(t, P)-K\left(t, P_{n-1}\right)\right) \\
& +\frac{2 \rho}{(2-\rho) M(\rho)} \int_{0}^{t}\left(K(y, P)-K\left(y, P_{n-1}\right)\right) d y \| \\
\leq & \frac{2(1-\rho)}{(2-\rho) M(\rho)}\left\|\left(K(t, P)-K\left(t, P_{n-1}\right)\right)\right\| \\
& +\frac{2 \rho}{(2-\rho) M(\rho)} \int_{0}^{t}\left\|\left(K(y, P)-K\left(y, P_{n-1}\right)\right)\right\| d y \\
\leq & \frac{2(1-\rho)}{(2-\rho) M(\rho)} \gamma_{1}\left\|P-P_{n-1}\right\| \\
& +\frac{2 \rho}{(2-\rho) M(\rho)} \gamma_{1}\left\|P-P_{n-1}\right\| t .
\end{aligned}
$$

On using this process recursively, it yields

$$
\left\|B_{n}(t)\right\| \leq\left(\frac{2(1-\rho)}{(2-\rho) M(\rho)}+\frac{2 \rho}{(2-\rho) M(\rho)} t\right)^{n+1} \gamma_{1}^{n+1} \alpha
$$


Now taking the limit on equation (31) as $n$ tends to infinity, we get

$$
\left\|B_{n}(t)\right\| \rightarrow 0
$$

Similarly, we get

$$
\left\|C_{n}(t)\right\| \rightarrow 0, \quad\left\|D_{n}(t)\right\| \rightarrow 0, \quad\left\|E_{n}(t)\right\| \rightarrow 0 \quad \text { and } \quad\left\|F_{n}(t)\right\| \rightarrow 0
$$

Hence existence is verified.

Now, we prove the uniqueness of a system of solutions of equation (9).

Let there exist another system of solutions of (9), $P_{1}(t), L_{1}(T), S_{1}(t), Q_{1}(t)$ and $R_{1}(t)$; then

$$
\begin{aligned}
P(t)-P_{1}(t)= & \frac{2(1-\rho)}{(2-\rho) M(\rho)}\left(K_{1}(t, P)-K_{1}\left(t, P_{1}\right)\right) \\
& +\frac{2 \rho}{(2-\rho) M(\rho)} \int_{0}^{t}\left(K_{1}(y, P)-K_{1}\left(y, P_{1}\right)\right) d y .
\end{aligned}
$$

Applying the norm on equation (32), we get

$$
\begin{aligned}
\left\|P(t)-P_{1}(t)\right\| \leq & \frac{2(1-\rho)}{(2-\rho) M(\rho)}\left\|K_{1}(t, P)-K_{1}\left(t, P_{1}\right)\right\| \\
& +\frac{2 \rho}{(2-\rho) M(\rho)} \int_{0}^{t}\left\|\left(K_{1}(y, P)-K_{1}\left(y, P_{1}\right)\right)\right\| d y .
\end{aligned}
$$

By employing the Lipschitz conditions of the kernel, we have

$$
\left\|P(t)-P_{1}(t)\right\| \leq \frac{2(1-\rho)}{(2-\rho) M(\rho)} \gamma_{1}\left\|P(t)-P_{1}(t)\right\|+\frac{2 \rho}{(2-\rho) M(\rho)} \gamma_{1} t\left\|P(t)-P_{1}(t)\right\|
$$

It gives

$$
\left\|P(t)-P_{1}(t)\right\|\left(1-\frac{2(1-\rho)}{(2-\rho) M(\rho)} \gamma_{1}-\frac{2 \rho}{(2-\rho) M(\rho)} \gamma_{1} t\right) \leq 0
$$

Theorem 4.3 The system of equations (9) has a unique system of solutions if the following condition holds:

$$
\left(1-\frac{2(1-\rho)}{(2-\rho) M(\rho)} \gamma_{1}-\frac{2 \rho}{(2-\rho) M(\rho)} \gamma_{1} t\right) \geq 0
$$

Proof If the condition (36) holds, then

$$
\left\|P(t)-P_{1}(t)\right\|\left(1-\frac{2(1-\rho)}{(2-\rho) M(\rho)} \gamma_{1}-\frac{2 \rho}{(2-\rho) M(\rho)} \gamma_{1} t\right) \leq 0,
$$

then we have

$$
\left\|P(t)-P_{1}(t)\right\|=0 .
$$


Then we get

$$
P(t)=P_{1}(t)
$$

Proceeding in the same way, we have

$$
\begin{aligned}
& L(t)=L_{1}(t), \\
& S(t)=S_{1}(t), \\
& Q(t)=Q_{1}(t), \\
& R(t)=R_{1}(t) .
\end{aligned}
$$

Therefore, we verified the uniqueness of the system of solutions of equations (9).

\section{Numerical results and discussions}

In this portion, we discuss the numerical simulations of the solutions of system of equations (9) as a function of time for distinct values of $\rho$. The numerical results are obtained by an iterative perturbation method and the Padè approximation [28]. We utilize the values of the parameters $a=0.04, b=0.23, c=0.3, d=0.2, e=0.4$ and $f=0.25$. The initial conditions are given by $P(0)=0.60301, L(0)=0.24000, S(0)=0.10628, Q(0)=0.03260$ and $R(0)=0.01811$. Figures 1-5, $P(t), L(t), S(t), Q(t)$ and $R(t)$ are investigated with respect to different values of $\rho$. Besides Figure 6 the displacement for smoking model is shown when $\rho=1$. From Figure 1 we can observe that the number of potential smokers $(P)$ increases with time and as the value of $\rho$ decreases the number of potential smokers $(P)$ decreases. From Figure 2 we can see that the number of occasional smokers $(L)$ increases with time and as the value of $\rho$ decreases, the number of occasional smokers $(L)$ decreases. From Figure 3 we can analyze that the number of heavy smokers $(S)$ decreases with time and as the value of $\rho$ decreases the number of heavy smokers $(S)$ increases. From Figure 4, we can observe that the number of temporary quitters $(Q)$ initially increases with time and with increasing the values of $\rho$, but after some time the behavior is opposite. From Figure 5 we can observe that the number of permanent quitters $(R)$ increases with time and as the

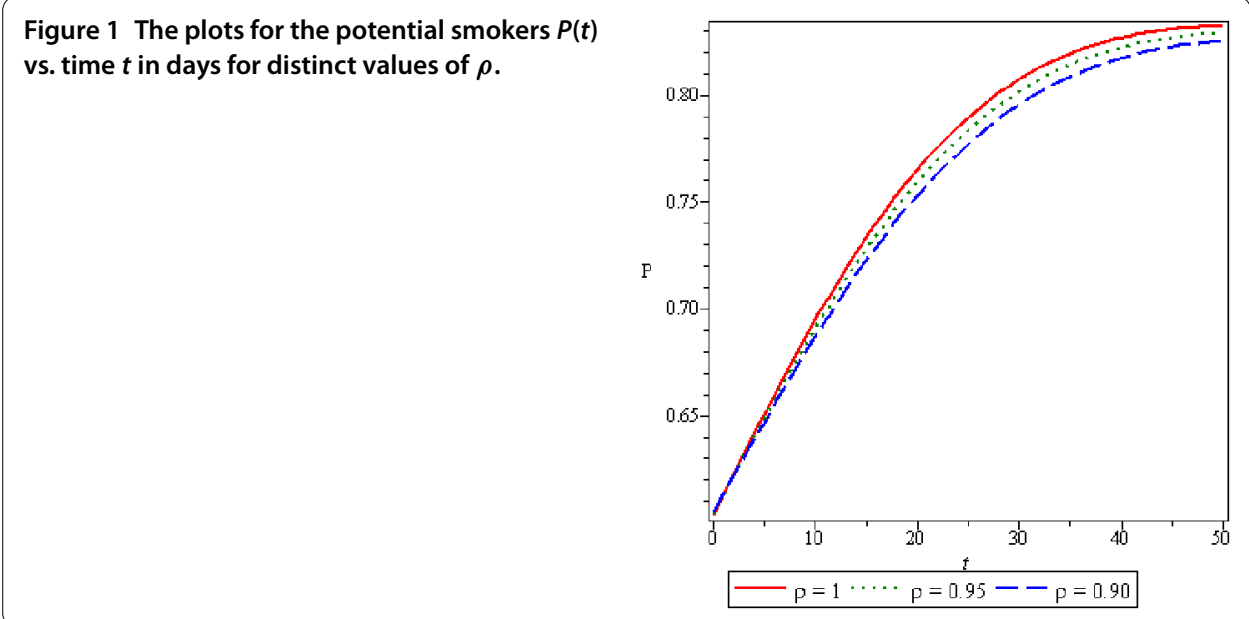




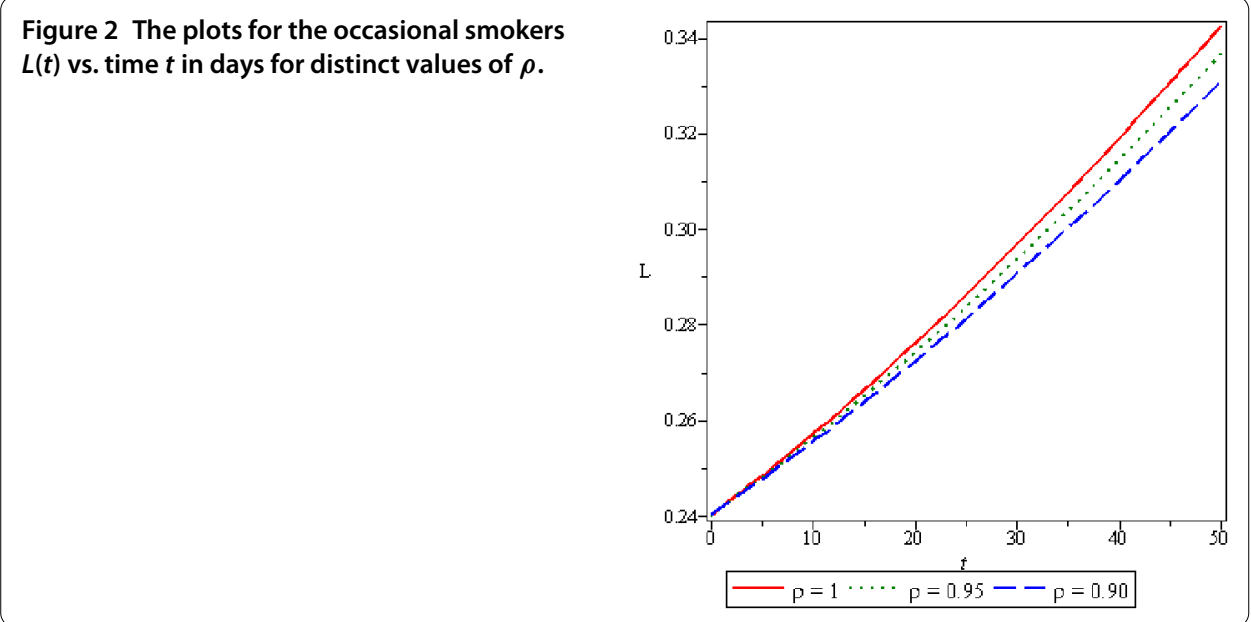

Figure 3 The plots for the heavy smokers $S(t)$ vs. time $t$ in days for distinct values of $\rho$.

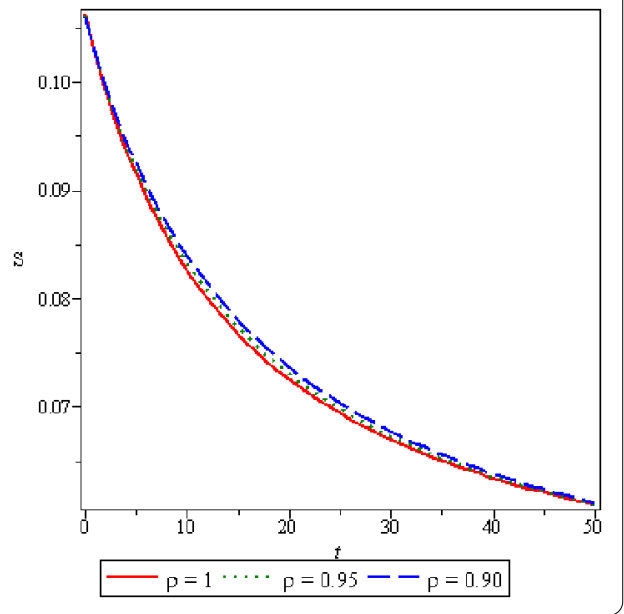

Figure 4 The plots for the temporary quitters $Q(t)$ vs. time $t$ in days for distinct values of $\rho$.

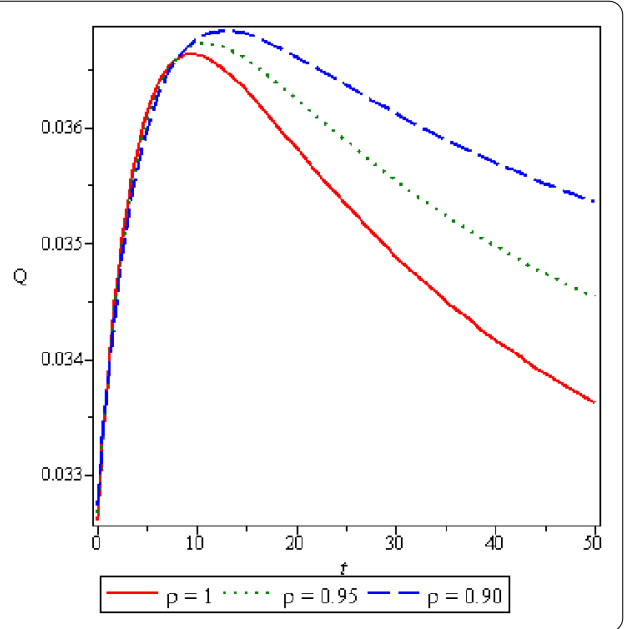




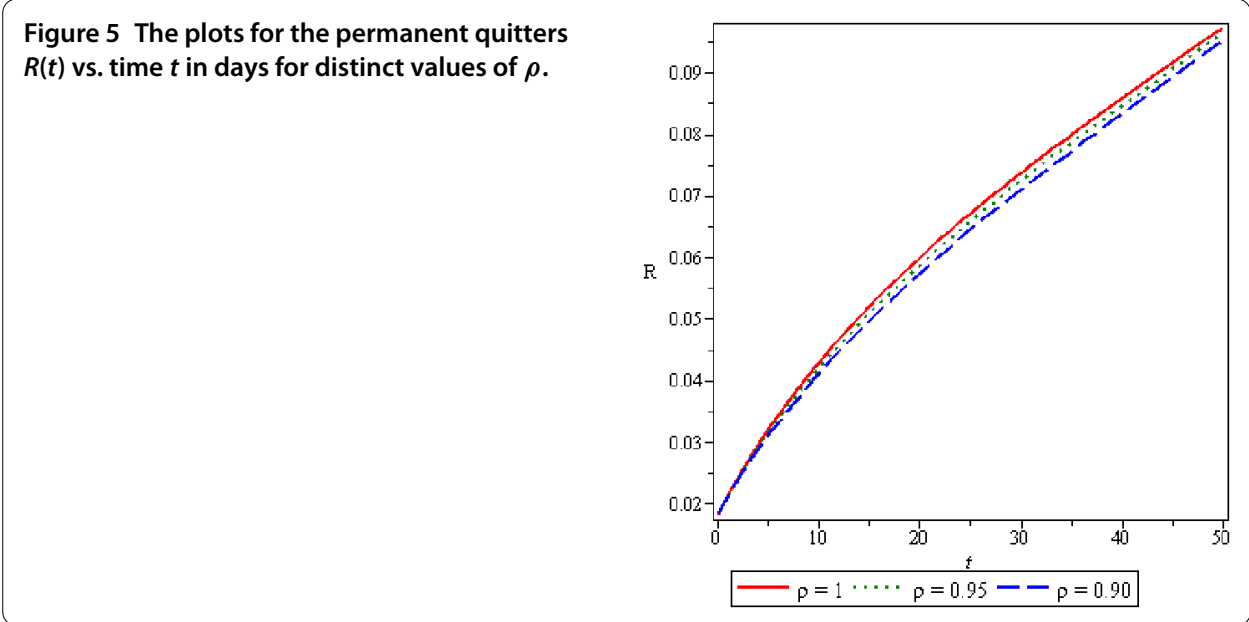

Figure 6 The displacement for the smoking model when $\rho=1$.

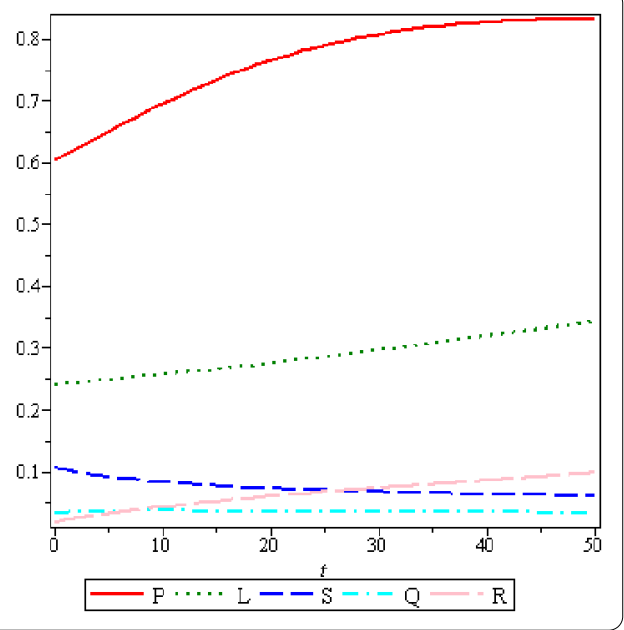

Table 1 Comparison between standard derivative, Caputo fractional derivatives and Caputo-Fabrizio fractional derivative for the potential smokers $P(t)$

\begin{tabular}{llll}
\hline $\boldsymbol{t}$ & $\begin{array}{l}\text { Standard derivative } \\
(\boldsymbol{\rho}=\mathbf{1})\end{array}$ & $\begin{array}{l}\text { Caputo derivative } \\
(\boldsymbol{\rho}=\mathbf{0 . 9 5})\end{array}$ & $\begin{array}{l}\text { Caputo-Fabrizio derivative } \\
(\boldsymbol{\rho}=\mathbf{0 . 9 5})\end{array}$ \\
\hline 1 & 0.6124398619 & 0.6126334075 & 0.6124401378 \\
2 & 0.6218771047 & 0.6216116071 & 0.6214032210 \\
3 & 0.6312904730 & 0.6303312432 & 0.6303433323 \\
4 & 0.6406487116 & 0.6388461359 & 0.6392336738 \\
5 & 0.6499205647 & 0.6471669258 & 0.6480474489 \\
\hline
\end{tabular}

value of $\rho$ decreases the number of permanent quitters $(R)$ decreases. From Figure 6 we can determine the displacement for the smoking model when $\rho=1$.

Tables 1-5 present the comparative study between the standard derivative, Caputo derivatives and Caputo-Fabrizio derivative. It can easily be observed from Tables 1-5 that the Caputo-Fabrizio fractional derivative shows the new nature compared to the standard derivative and Caputo fractional derivative. The graphical representations show that the model depends notably on the fractional order. Figures 1-5 and Tables 1-5 show the clear difference at different values of $\rho$. The model describes a new characteristic at the fractional values of $\rho$ that was unobserved when modeling at $\rho=1$. 
Table 2 Comparison between standard derivative, Caputo fractional derivatives and Caputo-Fabrizio fractional derivative for the occasional smokers $L(t)$

\begin{tabular}{llll}
\hline $\boldsymbol{t}$ & $\begin{array}{l}\text { Standard derivative } \\
(\boldsymbol{\rho}=\mathbf{1})\end{array}$ & $\begin{array}{l}\text { Caputo derivative } \\
(\boldsymbol{\rho}=\mathbf{0 . 9 5})\end{array}$ & $\begin{array}{l}\text { Caputo-Fabrizio derivative } \\
(\boldsymbol{\rho}=\mathbf{0 . 9 5})\end{array}$ \\
\hline 1 & 0.2416136687 & 0.2416475729 & 0.2416146612 \\
2 & 0.2432477561 & 0.2432024968 & 0.2431675083 \\
3 & 0.2449020824 & 0.2447359790 & 0.2447386087 \\
4 & 0.2465764697 & 0.2462618874 & 0.2463278099 \\
5 & 0.2482707411 & 0.2477865184 & 0.2479349601 \\
\hline
\end{tabular}

Table 3 Comparison between standard derivative, Caputo fractional derivatives and Caputo-Fabrizio fractional derivative for the heavy smokers $S(t)$

\begin{tabular}{llll}
\hline $\boldsymbol{t}$ & $\begin{array}{l}\text { Standard derivative } \\
(\boldsymbol{\rho}=\mathbf{1})\end{array}$ & $\begin{array}{l}\text { Caputo derivative } \\
(\boldsymbol{\rho}=\mathbf{0 . 9 5})\end{array}$ & $\begin{array}{l}\text { Caputo-Fabrizio derivative } \\
(\boldsymbol{\rho}=\mathbf{0 . 9 5})\end{array}$ \\
\hline 1 & 0.1026239640 & 0.1025641835 & 0.1026437765 \\
2 & 0.09937450414 & 0.09948799415 & 0.09956153608 \\
3 & 0.09646354464 & 0.09680263691 & 0.09678199000 \\
4 & 0.09382300970 & 0.09440789173 & 0.09424677174 \\
5 & 0.09138482355 & 0.09222574223 & 0.09189751483 \\
\hline
\end{tabular}

Table 4 Comparison between standard derivative, Caputo fractional derivatives and Caputo-Fabrizio fractional derivative for the temporary quitters $Q(t)$

\begin{tabular}{llll}
\hline $\boldsymbol{t}$ & $\begin{array}{l}\text { Standard derivative } \\
(\boldsymbol{\rho}=\mathbf{1})\end{array}$ & $\begin{array}{l}\text { Caputo derivative } \\
(\boldsymbol{\rho}=\mathbf{0 . 9 5})\end{array}$ & $\begin{array}{l}\text { Caputo-Fabrizio derivative } \\
(\boldsymbol{\rho}=\mathbf{0 . 9 5})\end{array}$ \\
\hline 1 & 0.03376048059 & 0.03377405752 & 0.03374711339 \\
2 & 0.03464368108 & 0.03460575042 & 0.03458444010 \\
3 & 0.03531175962 & 0.03521909598 & 0.03523042414 \\
4 & 0.03582687433 & 0.03568394356 & 0.03573835831 \\
5 & 0.03625118333 & 0.03606106299 & 0.03616153544 \\
\hline
\end{tabular}

Table 5 Comparison between standard derivative, Caputo fractional derivatives and Caputo-Fabrizio fractional derivative for the permanently quitters $R(t)$

\begin{tabular}{llll}
\hline $\boldsymbol{t}$ & $\begin{array}{l}\text { Standard derivative } \\
(\boldsymbol{\rho}=\mathbf{1})\end{array}$ & $\begin{array}{l}\text { Caputo derivative } \\
(\boldsymbol{\rho}=\mathbf{0 . 9 5})\end{array}$ & $\begin{array}{l}\text { Caputo-Fabrizio derivative } \\
(\boldsymbol{\rho}=\mathbf{0 . 9 5})\end{array}$ \\
\hline 1 & 0.02113719363 & 0.02107624005 & 0.02112963057 \\
2 & 0.02400835647 & 0.02369345384 & 0.02385493816 \\
3 & 0.02674146144 & 0.02611744835 & 0.02645645955 \\
4 & 0.02935448146 & 0.02839320512 & 0.02894960428 \\
5 & 0.03186538945 & 0.03055013348 & 0.03134978188 \\
\hline
\end{tabular}

\section{Concluding remarks}

In this paper, the smoking model is analyzed with the Caputo-Fabrizio derivative and makes use of the utilities of fractional calculus. To demonstrate the existence and uniqueness of a system of solutions the fixed point theorem is discussed. By employing an iterative perturbation method the special solution is obtained for the fractional order model. To present the effect of fractional order some numerical simulations are performed. By simulation it is clear that when $\rho$ tends to 1 the CF derivative shows a more interesting behavior. Hence, it can be concluded that the newly fractional derivative is very important for modeling real world problems. 
Competing interests

The authors declare that they have no competing interests.

\section{Authors' contributions}

JS, DK, MAQ and DB designed the study, developed the methodology, collected the data, performed the analysis, and wrote the manuscript. All authors read and approved the final manuscript.

\section{Author details}

'Department of Mathematics, JECRC University, Jaipur, Rajasthan 303905, India. ${ }^{2}$ Department of Mathematics, College of Science, King Saud University, Riyadh, 11495, Saudi Arabia. ${ }^{3}$ Department of Mathematics, Faculty of Arts and Sciences, Cankaya University, Eskisehir Yolu 29. Km, Yukarıyurtcu Mahallesi Mimar Sinan Caddesi No: 4, Etimesgut, 06790, Turkey.

${ }^{4}$ Institute of Space Sciences, Magurele-Bucharest, Romania.

\section{Acknowledgements}

The authors extend their appreciation to the International Scientific Partnership Program ISPP at King Saud University for funding this research work through ISPP\# 63.

\section{Publisher's Note}

Springer Nature remains neutral with regard to jurisdictional claims in published maps and institutional affiliations.

\section{Received: 8 December 2016 Accepted: 9 March 2017 Published online: 23 March 2017}

\section{References}

1. Erturk, VS, Zaman, G, Momani, S: A numeric analytic method for approximating a giving up smoking model containing fractional derivatives. Comput. Math. Appl. 64, 3068-3074 (2012)

2. Zaman, G: Optimal campaign in the smoking dynamics. Comput. Math. Methods Med. 2011, Article ID 163834 (2011).

3. Zaman, G: Qualitative behavior of giving up smoking models. Bull. Malays. Math. Soc. 34, 403-415 (2011)

4. Lubin, JL, Caporaso, ZE: Cigarette smoking and lung cancer: modeling total exposure and intensity. Cancer Epidemiol. Biomark. Prev. 15, 517-523 (2006)

5. Garsow, CC, Salivia, GJ, Herrera, AR: Mathematical Models for the Dynamics of Tobacoo use, recovery and relapse. Technical Report Series BU-1505-M, Cornell University, UK (2000)

6. Sharomi, O, Gumel, AB: Curtailing smoking dynamics: a mathematical modeling approach. Appl. Math. Comput. 195, 475-499 (2008)

7. Zeb, A, Chohan, I, Zaman, G: The homotopy analysis method for approximating of giving up smoking model in fractional order. Appl. Math. 3, 914-919 (2012)

8. Alkhudhari, Z, Al-Sheikh, S, Al-Tuwairqi, S: Global dynamics of a mathematical model on smoking. Appl. Math. 2014, Article ID 847075 (2014)

9. Khalid, M, Khan, FS, lqbal, A: Perturbation-iteration algorithm to solve fractional giving up smoking mathematical model. Int. J. Comput. Appl. 142, 1-6 (2016)

10. Caputo, M: Elasticita e Dissipazione. Zanichelli, Bologna (1969)

11. Baleanu, D, Guvenc, ZB, Machado, JAT: New Trends in Nanotechnology and Fractional Calculus Applications. Springer, Dordrecht (2010)

12. Kilbas, AA, Srivastava, HM, Trujillo, JJ: Theory and Applications of Fractional Differential Equations. Elsevier, Amsterdam (2006)

13. Bulut, H, Baskonus, HM, Belgacem, FBM: The analytical solutions of some fractional ordinary differential equations by Sumudu transform method. Abstr. Appl. Anal. 2013, Article ID 203875 (2013).

14. Atangana, A, Alkahtani, BT: Analysis of the Keller-Segel model with a fractional derivative without singular kernel. Entropy 17, 4439-4453 (2015)

15. Atangana, A, Alkahtani, BT: Analysis of non-homogenous heat model with new trend of derivative with fractional order. Chaos Solitons Fractals 89, 566-571 (2016)

16. Singh, J, Kumar, D, Kilichman, A: Numerical solutions of nonlinear fractional partial differential equations arising in spatial diffusion of biological populations. Abstr. Appl. Anal. 2014, Article ID 535793 (2014).

17. Kumar, D, Singh, J, Baleanu, D: A hybrid computational approach for Klein-Gordon equations on Cantor sets. Nonlinear Dyn. (2016). doi:10.1007/s11071-016-3057-x

18. Singh, J, Kumar, D, Swroop, R: Numerical solution of time- and space-fractional coupled Burgers equations via homotopy algorithm. Alex. Eng. J. 55, 1753-1763 (2016)

19. Kumar, D, Singh, J, Baleanu, D: Numerical computation of a fractional model of differential-difference equation. J. Comput. Nonlinear Dyn. 11, 061004 (2016)

20. Area, I, Batarfi, H, Losada, J, Nieto, JJ, Shammakh, W, Torres, A: On a fractional order Ebola epidemic model. Adv. Differ. Equ. (2015). doi:10.1186/s13662-015-0613-5

21. Carvalho, A, Pinto, CMA: A delay fractional order model for the co-infection of malaria and HIV/AIDS. Int. J. Dyn. Control (2016). doi:10.1007/s40435-016-0224-3

22. Huang, LL, Baleanu, D, Wu, GC, Zeng, SD: A new application of the fractional logistic map. Rom. J. Phys. 61, 1172-1179 (2016)

23. Ma, M, Baleanu, D, Gasimov, YS, Yang, XJ: New results for multidimensional diffusion equations in fractal dimensional space. Rom. J. Phys. 61, 784-794 (2016)

24. Kumar, D, Singh, J, Qurashi, MA, Baleanu, D: Analysis of logistic equation pertaining to a new fractional derivative with non-singular kernel. Adv. Mech. Eng. 9(2), 1-8 (2017)

25. Kumar, D, Singh, J, Baleanu, D: Modified Kawahara equation within a fractional derivative with non-singular kernel. Therm. Sci. (2017). doi:10.2298/TSCI160826008K 
26. Caputo, M, Fabrizio, M: A new definition of fractional derivative without singular kernel. Prog. Fract. Differ. Appl. 1, 73-85 (2015)

27. Losada, J, Nieto, J: Properties of the new fractional derivative without singular kernel. Prog. Fract. Differ. Appl. 1, 87-92 (2015)

28. Boyd, JP: Padè approximants algorithm for solving nonlinear ordinary differential equation boundary value problems on an unbounded domain. Comput. Phys. 11, 299-303 (1997)

Submit your manuscript to a SpringerOpen ${ }^{\circ}$ journal and benefit from:

- Convenient online submission

- Rigorous peer review

- Immediate publication on acceptance

- Open access: articles freely available online

- High visibility within the field

- Retaining the copyright to your article

Submit your next manuscript at $\boldsymbol{s p r i n g e r o p e n . c o m ~}$ 